\title{
Segmental duplication density decrease with distance to human-mouse breaks of synteny
}

\author{
Jesus Sainz ${ }^{*}, 1$, Pavol Rovensky ${ }^{1}$, Sigurjon A. Gudjonsson ${ }^{1}$, Gudmar Thorleifsson ${ }^{1}$, \\ Kari Stefansson $^{1,2}$ and Jeffrey R. Gulcher ${ }^{1,2, *}$ \\ ${ }^{1}$ deCODE Genetics, Reykjavik, Iceland
}

Segmental duplications are large genomic segments of recent origin and nearly identical sequence. Segmental duplications account for up to $5 \%$ of the human genome and they are often involved in genomic rearrangements and human disease. We developed a rapid computational method to characterize segmental duplications in the mouse and the human genomes according to four sequence assemblies for each species. Segmental duplication content in the mouse genome assemblies has largely changed over the four releases (from 0.2 to $1.2 \%, 4.5$ and $3.0 \%$ ), while in the four human assemblies duplication content was 4.8, 3.5, 3.7 and $3.7 \%$, respectively. This suggests that cataloguing and assembling duplications has been challenging in both genomes and any interpretation of comparative analyses of duplication content must keep this in perspective to avoid artifacts. Human and mouse segmental duplications are more frequent than expected in regions where there is a syntenic discontinuity and the duplication content in syntenic regions decreases significantly with distance from breakpoints of synteny. These observations indicate that in mouse and human the frequency of segmental duplications is strongly correlated with distance to human and mouse syntenic breaks or the most dynamic regions in evolution.

European Journal of Human Genetics (2006) 14, 216-221. doi:10.1038/sj.ejhg.5201534; published online 23 November 2005

Keywords: segmental duplications; synteny; evolution

\section{Introduction}

For some time, whole-genome duplications have been proposed as a model of evolution. ${ }^{1}$ More recently, segmental duplications have been shown to represent a large proportion of the human genome (up to 5\% for some analysis) and have been characterized as an important feature of genome organization. ${ }^{2-6}$ There is much evidence that implicate segmental duplications as one of the molecular mechanisms that lead to human diseases ${ }^{7-11}$ and that associate duplicated segments with genome

*Correspondence: Dr J Sainz or Dr JR Gulcher, deCODE Genetics, Sturlugotu 8, IS-101 Reykjavik, Iceland. Tel: + 354570 1946;

Fax: + 354570 1903;

E-mail: sainz@decode.is or Jeffrey.gulcher@decode.is

${ }^{2}$ These authors contributed equally to this work

Received 4 January 2005; revised 29 August 2005; accepted 27 September 2005; published online 23 November 2005 evolution. ${ }^{12-14}$ Recent findings of a high rate of gene conversion in palindromic sequences of human and ape chromosome $\mathrm{Y}$ suggest that gene conversion is a more frequent event than previously suspected, particularly in palindromic and duplicated sequences. ${ }^{15,16}$ This rapidly increasing body of data supports the notion that duplications play a very important role in the dynamics of genomic change.

Human chromosome 19 was the first chromosome analyzed with respect to duplications and human-mouse synteny and showed colocalization of duplicated genes and some breakpoints of synteny. ${ }^{17}$ The first genome-wide analysis of duplications and human-mouse synteny compared human genome assembly build 30 with the mouse assembly MGSCv3. ${ }^{18}$ The analysis showed dramatic enrichment of duplications at breakpoints of synteny in the human genome itself. They also analyzed the mouse $\mathrm{X}$ 
chromosome from build 30 and confirmed that there was an increase in duplication but did not provide data for the mouse autosomes. Our study extends the previous studies by (1) using more updated sequence assemblies for mouse and human, (2) comparing four successive assemblies for each to ensure that the observations were not artifacts of sequence assembly errors, and (3) defining enrichment of duplications in relation to distance to the breaks of synteny.

\section{Materials and methods}

\section{Genomic assemblies and synteny}

All genomic sequence assemblies and syntenic maps were downloaded from the University of California at Santa Cruz (UCSC) Genome Bioinformatics. Pericentromeric regions were defined by adding $1 \mathrm{Mb}$ to each side of the intervals annotated by UCSC Genome Bioinformatics as centromeric and their adjacent heterochromatic intervals in the 'chromAgp' files. Telomeric regions were defined as the $2 \mathrm{Mb}$ at the beginning (except in the acrocentric chromosomes) and the $2 \mathrm{Mb}$ at the end of the chromosomal sequences.

\section{Detection of segmental duplications}

We have developed an annotation package of programs and scripts to detect genomic segmental duplications. The package utilizes BLAT $^{19}$ as the alignment algorithm and allows the construction of segmental duplication detection in large genomes in a quick and efficient manner. The simplest model to detect segmental duplications would be to align the whole genome against itself but the current algorithms and computer memory available are limited to a much smaller query sequence sizes. Hence, we decided to divide the task into steps, which can be executed with moderate computing power using generally accepted tools and algorithms for alignment. The package cuts the genomic sequence into $1 \mathrm{~kb}$ consecutive segments, executes the alignment algorithm and analyzes the resulting alignments to define the duplications. We selected BLAT as the alignment program to use because it is fast and has the advantage that the masking of the DNA for high copy repeats is not necessary.

\section{Analysis of the alignments to detect duplications}

The identity of alignment is defined as 100 (matches/query size) (\%). Query size is $1 \mathrm{~kb}$. Alignments that are not selfhits and have a minimum identity of $90 \%$ are selected. Only blocks of the alignment that are located within a $1000 \mathrm{bp}$ window of the target are used. A recursive procedure assembles the duplicated genomic intervals for all queries using the following criteria:

a. The minimum length of the duplication is $5000 \mathrm{bp}$.

b. The total length of the gaps (in the target or the query) is less than $5 \%$ of the duplication.

\section{Post-processing}

Duplication intervals overlapping a minimum of $90 \%$ of their length with a single high-copy repetitive element are considered false positives and removed. A duplication interval is defined as an interval created by the set of overlapping or adjacent query sequences that constitute the duplication map. Duplications are defined as a query interval that has homology with a corresponding target interval. All the analyses in this manuscript use data from the query interval.

\section{Statistical methods}

We tested the significance of the observed difference in the number of direct versus inverted duplications and in the number of intrachromosomal versus interchromosomal duplications using a $\chi^{2}$ test. The difference in the average identity and average size of inter-versus intrachromosomal duplication intervals was tested using a randomization procedure, that is, randomly interchanging elements in the two groups. This procedure was repeated 100000 times and the $P$-value calculated as the fraction of randomization tests that yielded a difference in the average identity or average size that was equal or greater to the observed differences. All $P$-values presented are two-sided.

\section{Results \\ Human segmental duplications}

The criterion used to construct the duplication maps were set to a minimum of $5 \mathrm{~kb}$ duplication and $90 \%$ identity for each of the $1 \mathrm{~kb}$ fragments. The human segmental duplication maps were constructed using the National Center for Biotechnology Information (NCBI) build 31, NCBI build 33, NCBI build 34 and NCBI build 35 genomic assemblies. We will refer from now to the latest assembly, build 35, unless otherwise stated. Using an identity threshold of $90 \%$, we detected segmental duplications in 3119 human genomic intervals spanning $105.250 \mathrm{Mb}$ (3.7\% of the genome) with an average identity of $96.5 \%$ (Table 1). These intervals can include one or more segmental duplications.

The largest duplicated interval in human spans $1.5 \mathrm{Mb}$ on chromosome $\mathrm{Y}$ and the average duplicated interval is $33.7 \mathrm{~kb}$. Chromosomal distribution of duplications is not uniform, with chromosome $\mathrm{Y}$ and 9 having the greatest duplication content (53.0 and $9.0 \%$, respectively), and chromosome 3 having the least duplication content $(0.7 \%)$ (Figure 1a). Among the intrachromosomal duplications, inverted duplications are more frequent than direct duplications (54 versus $46 \%, P=1.3 \times 10^{-13}$ ) in build 35 . The ratio of inverted duplications versus direct duplications has increased in the finished assemblies, from 0.8 in build 31 and 1.0 in build 33 to 1.2 in build 34 and build 35 (Table 1). 
Table 1 Segmental duplications in four human genomic assemblies

\begin{tabular}{|c|c|c|c|c|}
\hline Human duplications & Build31 & Build33 & Build34 & Build35 \\
\hline Duplicated genome length (bp) & 136600000 & 99831000 & 104407000 & 105250000 \\
\hline Duplicated genome fraction (\%) & 4.80 & 3.52 & 3.67 & 3.69 \\
\hline Number of duplicons & 4486 & 2875 & 2960 & 3119 \\
\hline Average identity (\%) & 96.60 & 96.30 & 96.34 & 96.51 \\
\hline Average identity of intrachromosomal (\%) & 97.68 & 97.24 & 97.33 & 97.50 \\
\hline Average identity of interchromosomal (\%) & 95.45 & 95.42 & 95.50 & 95.61 \\
\hline Ratio intra/inter chromosomal (length) & 2.52 & 1.96 & 1.77 & 1.74 \\
\hline Ratio inverted/direct duplications (number) & 0.83 & 0.98 & 1.18 & 1.18 \\
\hline Largest duplicon length (bp) & 2829000 & 3028000 & 1526000 & 1526000 \\
\hline Average duplicon length (bp) & 30450 & 34723 & 35272 & 33744 \\
\hline Median duplicon length (bp) & 12000 & 13000 & 12000 & 12000 \\
\hline Average intrachromosomal duplicon length (bp) & 30720 & 35988 & 35084 & 33500 \\
\hline Average interchromosomal duplicon length (bp) & 22586 & 23102 & 24203 & 24163 \\
\hline
\end{tabular}
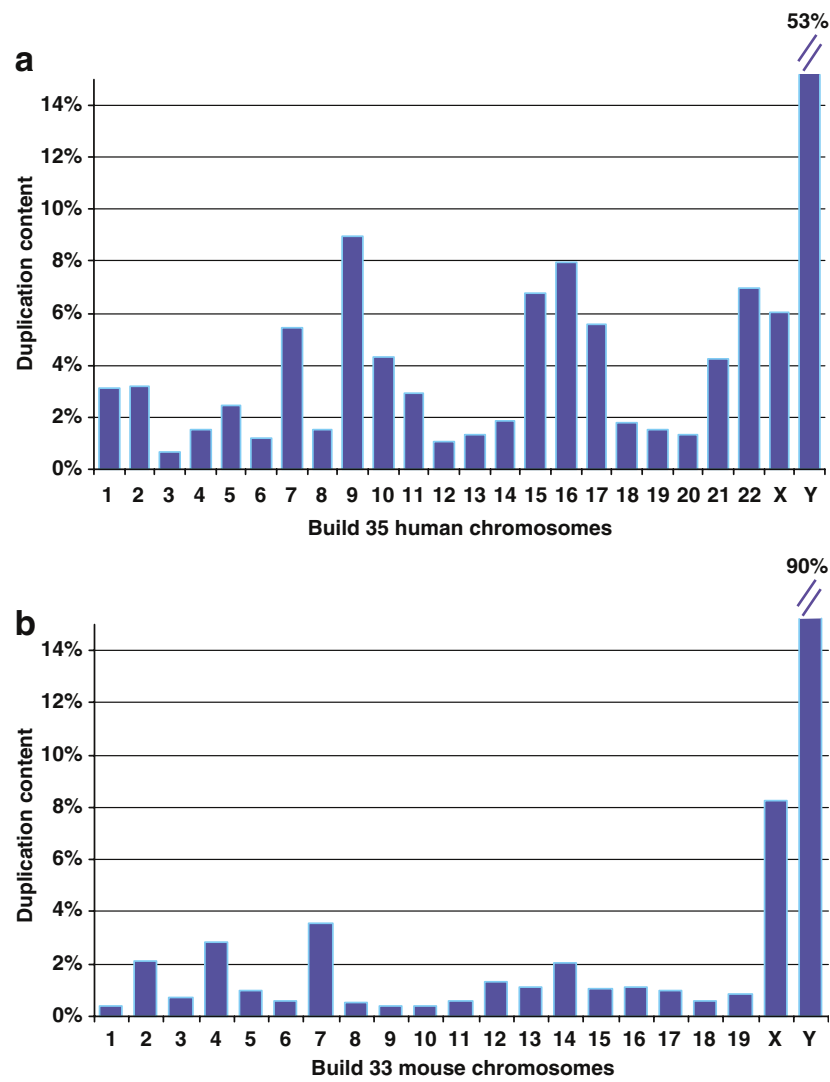

Figure 1 Duplication content of mouse and human genomes. (a) Human duplication content per chromosome. (b) Mouse duplication content per chromosome.

Intrachromosomal duplications are 1.7 times more abundant than interchromosomal duplications $\left(P<10^{-100}\right)$, have a higher average identity $(97.5 \%$ compared to $95.6 \%, P<0.00001)$, and have a larger average size ( $33.5 \mathrm{~Kb}$ compared to $24.2 \mathrm{~Kb}, P<0.00001)$. The ratio of intrachromosomal duplication versus interchromosomal duplication content has diminished in the consecutive assemblies (Table 1).

\section{Mouse segmental duplications}

The segmental duplication maps were constructed using the mouse assemblies MGSCv3, NCBI build 30, NCBI build 32 and NCBI build 33. We will refer from now on to the latest mouse assembly, build 33, unless indicated otherwise. The mouse duplications span a total of $74.2 \mathrm{Mb}$ or $2.98 \%$ of mouse genome. The number of duplicated intervals is 2807 and their average size is $26.4 \mathrm{~kb}$ with an average identity of $97.3 \%$. The largest duplication spans $564 \mathrm{~kb}$ on chromosome Y.

As in the human, chromosomal distribution of duplications in the mouse is not uniform, ranging from $0.38 \%$ on chromosome 10 to $8.2 \%$ on chromosome $\mathrm{X}$ and $90.2 \%$ on chromosome Y (Figure 1b), inverted duplications are more frequent than direct duplications (51.1 and 48.9\%, $P=3.4 \times 10^{-10}$ ) and the ratio of inverted versus direct duplications has increased in the two latest assemblies (Table 2).

As in human, intrachromosomal duplications are more abundant (6.45-fold more) than interchromosomal duplications $\left(P<10^{-100}\right)$, have a higher average identity $(97.3 \%$ compared to $96.9 \%, P<0.00001)$, and the intervals containing intrachromosomal duplications have a larger average size (30.6 versus $9.2 \mathrm{~kb}, P<0.00001)$. Intrachromosomal duplications are more abundant and their identity higher than interchromosomal duplications in all mouse assemblies but the ratio has fluctuated greatly (Table 2).

Analysis of mouse and human duplications show no sequence similarity as would be expected for duplications that have arisen since the lineage of these species separated 75 million years ago.

\section{Synteny and segmental duplications}

Long-range chromosomal organization in human and mouse is known to be similar and conserved syntenic 
Table 2 Segmental duplications in four mouse assemblies

\begin{tabular}{|c|c|c|c|c|}
\hline Mouse duplications & MGSCV3 & Build 30 & Build 32 & Build 33 \\
\hline Duplicated genome length (bp) & 4396000 & 28648000 & 111526000 & 74200000 \\
\hline Duplicated genome fraction (\%) & 0.18 & 1.19 & 4.47 & 2.98 \\
\hline Number of duplicons & 633 & 1879 & 5539 & 2807 \\
\hline Average identity (\%) & 96.39 & 97.07 & 97.16 & 97.26 \\
\hline Average identity of intrachromosomal (\%) & 96.41 & 97.86 & 97.53 & 97.31 \\
\hline Average identity of interchromosomal (\%) & 96.39 & 96.85 & 96.68 & 96.86 \\
\hline Ratio intra/inter chromosomal (length) & 1.23 & 5.86 & 2.85 & 6.45 \\
\hline Ratio inverted/direct duplications (number) & 0.60 & 0.37 & 0.94 & 1.05 \\
\hline Largest duplicon length (bp) & 114000 & 228000 & 430000 & 564000 \\
\hline Average duplicon length (bp) & 6944 & 15246 & 20134 & 26433 \\
\hline Median duplicon length (bp) & 6000 & 8000 & 10000 & 8000 \\
\hline Average intrachromosomal duplicon length (bp) & 7374 & 17315 & 21327 & 30620 \\
\hline Average interchromosomal duplicon length (bp) & 6282 & 7170 & 16238 & 9236 \\
\hline
\end{tabular}

regions have been reported..$^{2,3,20,21}$ To further investigate a possible role of segmental duplications in chromosomal rearrangement during speciation, we placed the locations of segmental duplications on human/mouse synteny maps generated by the UCSC. The latest syntenic map has high resolution and identifies 1111 regions of human synteny with mouse that account for $92.4 \%$ of the human sequence (here called 'syntenic genome'). The nonsyntenic intervals (here called 'nonsyntenic genome') account for 7.6\% of the human sequence. Human duplications are 7.2 times more frequent than expected by chance in genomic regions nonsyntenic with mouse: $55.1 \%$ of duplicated sequences locate in the $7.6 \%$ nonsyntenic genome. The human nonsyntenic genome spans $218.6 \mathrm{Mb}$ and $26.6 \%$ of it $(58.0 \mathrm{Mb})$ is comprised of segmental duplications. In contrast the syntenic genome spans $2650 \mathrm{Mb}$ with segmental duplications making up only $1.8 \%(47.2 \mathrm{Mb})$. However, analysis of the syntenic $50 \mathrm{~kb}$ adjacent to the breaks of synteny indicates that a higher fraction is duplicated: $17.5 \mathrm{Mb}$ of a total of $87.2 \mathrm{Mb}(20.1 \%)$. This indicates that not only the nonsyntenic regions but also the adjacent syntenic regions have a much higher frequency of duplications than the rest of the genome.

To determine if the duplication content is related to the distance from the junction of syntenic discontinuity, we analyzed genomic intervals on both sides of all junctions in the genome. The duplicated fraction of the syntenic genome at a distance to the junction greater than $100 \mathrm{~kb}$, from 100 to $50 \mathrm{~kb}$, from 50 to $20 \mathrm{~kb}$ and from 20 to $0 \mathrm{~kb}$ is $1.0,8.8,15.6$ and $25.0 \%$, respectively. Duplication content has a strong negative correlation with the distance to the breaks of synteny in the four intervals analyzed (distance as the median for each class of interval; correlation coefficient $=-0.82$ ). Duplication content of the nonsyntenic intervals from 0 to $20 \mathrm{~kb}$ and $>20 \mathrm{~kb}$ to the synteny junction is 24.7 and $27.0 \%$, respectively. These results show that the duplication content increases with the proximity to the nonsyntenic regions and reaches maximum values

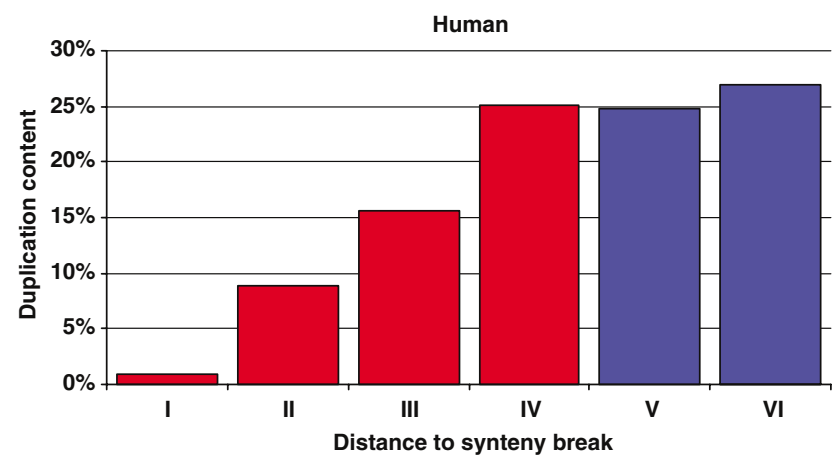

Figure 2 Duplication content in human build 35 according to the distance to synteny breaks with mouse. Blue bars represent nonsyntenic genome and red bars syntenic genome. Bar numbers: I. In synteny and at more than $100 \mathrm{~kb}$ to the junction. II. In synteny and from 100 to $50 \mathrm{~kb}$ to the junction. III. In synteny and from 50 to $20 \mathrm{~kb}$ to the junction. IV. In synteny and from 20 to $0 \mathrm{~kb}$ to the junction. V. In nonsynteny and from 0 to $20 \mathrm{~kb}$ to the junction. VI. In nonsynteny and at more than $20 \mathrm{~kb}$ to the junction.

in the internal part of the nonsyntenic region (Figure 2). The higher duplication content in the syntenic genome near the break of synteny junctions is observed for all the human chromosomes with the only exception of chromosome Y.

Analysis of the mouse synteny show, as in human, that duplications are 8.0 times more abundant than expected in sequences that are nonsyntenic with human: $63.4 \%$ of the duplications are in the $7.9 \%$ nonsyntenic mouse genome. The mouse nonsyntenic genome spans $202.9 \mathrm{Mb}$ and contains $47.1 \mathrm{Mb}$ of segmental duplications or $23.2 \%$ of its length while the syntenic genome spans $2366 \mathrm{Mb}$ with $27.1 \mathrm{Mb}$ of segmental duplications or $1.1 \%$ of the length. As in human, duplication density in mouse decreases with distance to the breaks of synteny. For the syntenic intervals at greater than $100 \mathrm{~kb}$, from 100 to $50 \mathrm{~kb}$, from 50 to $20 \mathrm{~kb}$ and from 20 to $0 \mathrm{~kb}$, the duplication fraction is $1.0,2.2,3.5$ and $4.9 \%$, respectively. As in human, the duplication 


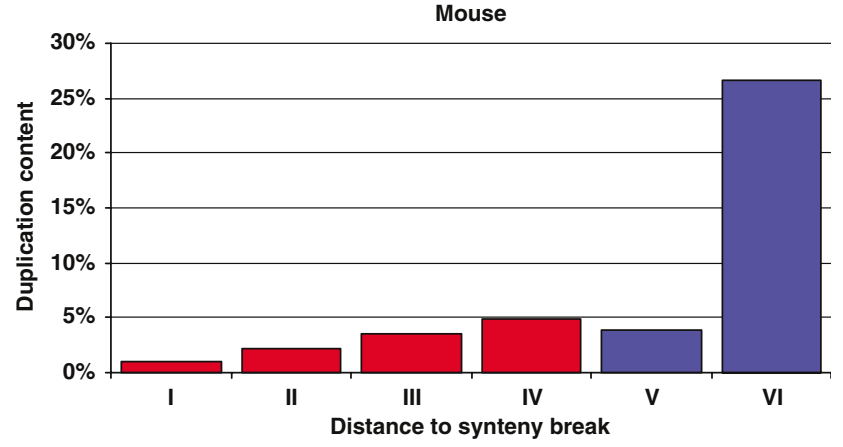

Figure 3 Duplication content in mouse build 33 according to the distance to synteny breaks with human. Blue bars represent nonsyntenic genome and red bars syntenic genome. Bar numbers: I. In synteny and at more than $100 \mathrm{~kb}$ to the junction. II. In synteny and from 100 to $50 \mathrm{~kb}$ to the junction. III. In synteny and from 50 to $20 \mathrm{~kb}$ to the junction. IV. In synteny and from 20 to $0 \mathrm{~kb}$ to the junction. V. In nonsynteny and from 0 to $20 \mathrm{~kb}$ to the junction. VI. In nonsynteny and at more than $20 \mathrm{~kb}$ to the junction.

content has a strong negative correlation with the distance to breaks of synteny of the four intervals analyzed (distance as the median for each class of interval; correlation coefficient $=-0.76$ ). For the nonsyntenic intervals from 0 to $20 \mathrm{~kb}$ and $>20 \mathrm{~kb}$ the duplicated fraction is 3.8 and $26.7 \%$, respectively (Figure 3 ). In mouse, we observe a much greater density of duplications in the nonsyntenic intervals at a distance of more than $20 \mathrm{~kb}$ of the syntenic regions than the density in the $20 \mathrm{~kb}$ intervals adjacent to the synteny/nonsynteny boundaries (Figure 3). This difference is not observed in human (Figure 2). It is not clear to us the reason for this difference.

We asked the question whether duplications in nonsyntenic intervals were clustered in pericentromeric or telomeric regions and we found that this was not the case: the percentage of all segmental duplications detected in nonsyntenic regions when we exclude the pericentromeric or telomeric regions in the analysis (51\% in human and $62 \%$ in mouse) is not very different from the percentage detected including these regions (55\% in human and 63\% in mouse).

We found large differences in segmental duplication content between the four mouse sequence assemblies analyzed. Even with the large range of variation in segmental duplication content for the mouse assemblies, duplication content in synteny breaks higher than expected by a random distribution was found in all mouse assemblies. Thus, duplications correlate with syntenic breaks. These results confirm previous studies that showed colocalization of duplicated genes and breakpoints of synteny, specifically in human chromosome $19^{17}$ and in the human genome in general by comparing human assembly build 30 and mouse assembly build $30 .{ }^{18}$ Our study shows that these observations hold up even in the most recent sequence assemblies and when performed at high resolution. We furthermore found that the duplica- tion prevalence shows a gradient that peaks at the breaks in synteny.

\section{Discussion}

A possible caveat could be that a large fraction of the breaks of synteny used might have been generated mainly by lack of sequence alignment between both genomes and do not reflect rearrangements originated in the evolution from an ancestral genome. We believe that our evidence supports that this is not the case. We analyzed the breaks of synteny and in at least $85 \%$ of the cases they corresponded to clear genomic rearrangements caused by breaks joining different chromosomes, by intrachromosomal inversions, or by intrachromosomal translocations. Restricting the analysis to the breaks of synteny clearly originated by genomic rearrangements indicates that their duplication content is similarly high than the one observed analyzing all breaks of synteny (26.9 versus $26.6 \%$ for all breaks in human and 22.0 versus $23.2 \%$ in mouse).

One may argue that the human genome that appears to be nonsyntenic to mouse, represents parts of the mouse genome yet to be sequenced or vice versa and that segmental duplications are more difficult to sequence. However, in our analyses of the four human genome sequence assemblies, we observe consistent results. Therefore, even if there are limitations in the mouse sequence assemblies that create collapses of real duplications and pseudo-duplications or other errors, it would be hard to support that these limitations generate the striking correlation to syntenic discontinuities, particularly when in the most recent and perfected assemblies the correlation becomes stronger.

We observe that even with large changes in duplication content from previous mouse assemblies to the latest one, the duplication content is always higher than expected at the breaks of synteny. In the latest mouse assembly (build $33)$, the duplication characteristics are more consistent with the ones in the finished human genome than it was in older mouse assemblies. The latest mouse assembly has a similar percentage of duplicated genome to that of the human, a higher content of duplications in the regions were the synteny breaks as in human and a higher duplication content near the breaks of synteny as in human. Also the average identity of interchromosomal duplications and intrachromosomal duplications is similar in the last mouse assembly and in the last human assembly. It is important to note that mouse build 32 and build 33 used a clone-based tiling path file method for the assembly, a more robust method to prevent the collapse of duplications than the whole genome shotgun sequence method used in the previous assemblies. ${ }^{22}$

We observe that inverted duplications are more frequent than direct duplications in human build 34 and build 35 
while they are similarly frequent in build 33 and they are less frequent in build 31 . The increase in the ratio inverted/ direct duplications is also observed in the last three mouse assemblies.

A common feature in all human and mouse assemblies analyzed is that intrachromosomal duplications had a higher identity than interchromosomal duplications. In the current assemblies the identity is $1.9 \%$ higher in human and $0.5 \%$ higher in mouse. Given that it is unlikely that intrachromosomal duplications are on average more recent than interchromosomal duplications, we think that molecular mechanisms such as gene conversion could help to preserve the sequence identity of duplications within the same chromosomes in a higher degree than between different chromosomes.

The common characteristics of segmental duplications in the most recent mouse and human assemblies, particularly if we consider that mouse and human duplication sequences do not share any significant homology, suggest that the mechanisms that generate and preserve segmental duplications in mouse and human have similar molecular bases and that these mechanisms are, at least in great extent, independent of the sequence content of the duplicated segment.

In human and in mouse, the duplication content of the syntenic genome increases with proximity to the junctions where the synteny breaks and the regions of discontinuity in the synteny have the highest content of duplications. It has been reported that rearranged chromosomes associate with an accelerated rate of evolution. ${ }^{23}$ Considering that segmental duplications tend to be located where mouse and human ancestral chromosomes have been rearranged, we can hypothesize that segmental duplications are a driver for genomic and chromosomal evolution in man and mouse.

\section{Acknowledgements}

We thank Dr Augustine Kong for helpful comments.

\section{References}

1 Ohno S, Wolf U, Atkin NB: Evolution from fish to mammals by gene duplication. Hereditas 1968; 59: 169-187.

2 Lander ES, Linton LM, Birren B et al: Initial sequencing and analysis of the human genome. Nature 2001; 409: 860-921.

3 Waterston RH, Lindblad-Toh K, Birney E et al: Initial sequencing and comparative analysis of the mouse genome. Nature 2002; 420: $520-562$.
4 Cheung VG, Nowak N, Jang W et al: Integration of cytogenetic landmarks into the draft sequence of the human genome. Nature 2001; 409: 953-958.

5 Bailey JA, Gu Z, Clark RA et al: Recent segmental duplications in the human genome. Science 2002; 297: 1003-1007.

6 Cheung J, Estivill X, Khaja R et al: Genome-wide detection of segmental duplications and potential assembly errors in the human genome sequence. Genome Biol 2003; 4: R25.

7 Gratacos M, Nadal M, Martin-Santos R et al: A polymorphic genomic duplication on human chromosome 15 is a susceptibility factor for panic and phobic disorders. Cell 2001; 106: 367-379.

8 Mazzarella R, Schlessinger D: Pathological consequences of sequence duplications in the human genome. Genome Res 1998; 8: $1007-1021$.

9 Emanuel BS, Shaikh TH: Segmental duplications: an 'expanding' role in genomic instability and disease. Nat Rev Genet 2001; 2: 791-800.

10 Stankiewicz P, Lupski JR: Genome architecture, rearrangements and genomic disorders. Trends Genet 2002; 18: 74-82.

11 Singleton $\mathrm{AB}$, Farrer $\mathrm{M}$, Johnson $\mathrm{J}$ et al: alpha-Synuclein locus triplication causes Parkinson's disease. Science 2003; 302: 841.

12 Samonte RV, Eichler EE: Segmental duplications and the evolution of the primate genome. Nat Rev Genet 2002; 3: 65-72.

13 Eichler EE: Recent duplication, domain accretion and the dynamic mutation of the human genome. Trends Genet 2001; 17: $661-669$.

14 Goidts V, Szamalek JM, Hameister H, Kehrer-Sawatzki H: Segmental duplication associated with the human-specific inversion of chromosome 18: a further example of the impact of segmental duplications on karyotype and genome evolution in primates. Hum Genet 2004; 115: 116-122.

15 Skaletsky H, Kuroda-Kawaguchi T, Minx PJ et al: The male-specific region of the human $\mathrm{Y}$ chromosome is a mosaic of discrete sequence classes. Nature 2003; 423: 825-837.

16 Rozen S, Skaletsky H, Marszalek JD et al: Abundant gene conversion between arms of palindromes in human and ape $\mathrm{Y}$ chromosomes. Nature 2003; 423: 873-876.

17 Dehal P, Predki P, Olsen AS et al: Human chromosome 19 and related regions in mouse: conservative and lineage-specific evolution. Science 2001; 293: 104-111.

18 Armengol L, Pujana MA, Cheung J, Scherer SW, Estivill X: Enrichment of segmental duplications in regions of breaks of synteny between the human and mouse genomes suggest their involvement in evolutionary rearrangements. Hum Mol Genet 2003; 12: 2201-2208.

19 Kent WJ: BLAT - the BLAST-like alignment tool. Genome Res 2002; 12: 656-664.

20 Bailey JA, Yavor AM, Viggiano L et al: Human-specific duplication and mosaic transcripts: the recent paralogous structure of chromosome 22. Am J Hum Genet 2002; 70: 83-100.

21 Gregory SG, Sekhon M, Schein J et al: A physical map of the mouse genome. Nature 2002; 418: 743-750.

22 She X, Jiang Z, Clark RA et al: Shotgun sequence assembly and recent segmental duplications within the human genome. Nature 2004; 431: 927-930.

23 Navarro A, Barton NH: Chromosomal speciation and molecular divergence - accelerated evolution in rearranged chromosomes. Science 2003; 300: 321-324. 